\title{
Escalation with Overdose Control Using Ordinal Toxicity Grades for Cancer Phase I Clinical Trials
}

\author{
Mourad Tighiouart, Galen Cook-Wiens, and André Rogatko \\ Samuel Oschin Comprehensive Cancer Institute, 8700 Beverly Boulevard, Los Angeles, CA 90048, USA \\ Correspondence should be addressed to Mourad Tighiouart, mourad.tighiouart@cshs.org
}

Received 29 June 2012; Accepted 10 September 2012

Academic Editor: Zhengjia Chen

Copyright (c) 2012 Mourad Tighiouart et al. This is an open access article distributed under the Creative Commons Attribution License, which permits unrestricted use, distribution, and reproduction in any medium, provided the original work is properly cited.

We extend a Bayesian adaptive phase I clinical trial design known as escalation with overdose control (EWOC) by introducing an intermediate grade 2 toxicity when assessing dose-limiting toxicity (DLT). Under the proportional odds model assumption of dose-toxicity relationship, we prove that in the absence of DLT, the dose allocated to the next patient given that the previously treated patient had a maximum of grade 2 toxicity is lower than the dose given to the next patient had the previously treated patient exhibited a grade 0 or 1 toxicity at the most. Further, we prove that the coherence properties of EWOC are preserved. Simulation results show that the safety of the trial is not compromised and the efficiency of the estimate of the maximum tolerated dose (MTD) is maintained relative to EWOC treating DLT as a binary outcome and that fewer patients are overdosed using this design when the true MTD is close to the minimum dose.

\section{Introduction}

Cancer phase I clinical trials are sequential designs enrolling late stage cancer patients who have exhausted standard treatment therapies [1]. For cytotoxic agents or combinations of biologic with cytotoxic drugs, the main objectives of these trials are to characterize treatmentrelated toxicities and estimate a dose level that is associated with a predetermined level of dose limiting toxicity (DLT). Such a dose is called maximum tolerated dose (MTD) or phase II dose. Specifically, the MTD, $\gamma$, is defined as the dose that is expected to produce DLT after one cycle of therapy in a specified proportion $\theta$ of patients:

$$
P(\text { DLT } \mid \text { Dose }=\gamma)=\theta \text {. }
$$

Although the definition of DLT depends on the cancer type and the agent under study, it is typically defined as a grade 3 or 4 nonhematologic and grade 4 hematologic toxicity for 
cytotoxic agents, see the National Cancer Institute (NCI) common toxicity criteria [2]. The value chosen for the target probability of DLT $\theta$ depends on the nature and severity of the DLT; it is set relatively high when the DLT is a transient, reversible, and nonfatal condition and low when it is lethal or life threatening.

Model-based designs for cancer phase I clinical trials have been studied extensively in the last two decades; see O'Quigley et al. [3], Gatsonis and Greenhouse [4], Durham and Flournoy [5], Korn et al. [6], Whitehead and Brunier [7], Whitehead [8], Babb et al. [9], Gasparini and Eisele [10], Mukhopadhyay [11], and Haines et al. [12]. In particular, the continual reassessment method (CRM) first proposed by O'Quigley et al. [3] is Bayesian outcome adaptive, and has been modified and extended by many authors; see, for example, Faries [13], Moller [14], Goodman et al. [15], O'Quigley and Shen [16], Piantadosi et al. [17], Cheung and Chappell [18], Storer [19], and Leung and Wang [20]. The attribute of the CRM is that at each stage of the trial, we seek a dose for which a Bayes estimate of the probability of DLT is closest to the target probability of DLT $\theta$. Escalation with overdose control (EWOC) originally proposed by Babb et al. [9] is another alternative Bayesian outcome adaptive design for dose finding in early phase cancer trials. Unlike CRM, its main feature is that at each stage of the trial, we seek a dose for which the posterior probability of exceeding the MTD $\gamma$ is bounded by a feasibility bound $\alpha$. Statistical properties of EWOC have been studied by Zacks et al. [21], Tighiouart et al. [22], and Tighiouart and Rogatko [23], and examples of phase I trials using EWOC can be found in [24-33]. Further literature review of statistical methods for dose finding in cancer phase I trials can be found in Ting [34] and Chevret [35].

The above methods allocate future doses based on a binary outcome of DLT of previously treated patients. Such designs may not be efficient in the sense that the dose recommended for the next patient is the same regardless whether the previously treated patient had no toxicity or had an intermediate grade 2 toxicity. The work we present in this manuscript is motivated by the ethical concern raised by clinical colleagues regarding dose escalation in the absence of DLT. Specifically, if the current patient experiences drug related grade 2 toxicity at the most, then the dose to be allocated to the next patient should not be as high as the one had the current patient experienced a maximum of grade 0 or 1 toxicity. We present a Bayesian outcome adaptive design which is an extension of EWOC by accommodating an intermediate grade 2 toxicity to the model. We use a proportional odds model to describe the dose-toxicities relationship and the design is termed EWOC proportional odds model, written as EWOC-POM. We show that the design satisfies the above ethical consideration without compromising the safety and efficiency of the trial. Furthermore, we show that the design maintains the coherence properties of EWOC.

Cancer phase I clinical trials designs taking into account all grades and types of toxicities have been proposed by many authors with the goal of improving the safety and efficiency of the trial, see Gordon and Willson [36], Wang et al. [37], Bekele and Thall [38], Yuan et al. [39], Potthoff and George [40], Bekele et al. [41], Van Meter et al. [42, 43], Iasonos et al. [44], Lee et al. [45], and Chen et al. [46]. Some of these methods use multivariate models for characterizing the different grades of toxicities as a function of dose while others summarize the information from all toxicity grades into a continuous score. Depending on the underlying scenarios, in general, adding multiple toxicity grades information to the model has little effect on the safety of the trial with a modest gain in the precision of the estimate of the MTD. Our contribution in this manuscript is motivated by the ethical constraint that dose escalation in the absence of DLT should be mitigated by the occurrence of an intermediate grade 2 toxicity. The model and design we propose is constructed in such a way as to maintain the main 
properties of EWOC while at the same time, satisfying those ethical considerations raised by our clinical colleagues.

The manuscript is organized as follows. In Section 2, we give a detailed description of the methodology and describe the trial design. In Section 3, we state and prove the ethical considerations and coherence properties of EWOC-POM. The simulation results of the design operating characteristics and comparison with EWOC design are included in Section 4. Section 5 contains some final remarks and discussion of practical implementations.

\section{Method}

\subsection{Model}

Let $G=0,1, \ldots, 4$ be the maximum grade of toxicity experienced by a patient by the end of one cycle of therapy and define DLT as a maximum of grade 3 or 4 toxicity. Let

$$
Y= \begin{cases}0 & \text { if } G=0 \text { or } 1 \\ 1 & \text { if } G=2 \\ 2 & \text { if } G=3 \text { or } 4\end{cases}
$$

We model the dose-toxicities relationship by assuming that

$$
P(Y \geq j \mid x)=F\left(\alpha_{j}+\beta x\right), \quad j=1,2
$$

where $F(\cdot)$ is a known strictly increasing cdf. This implies that $\alpha_{2} \leq \alpha_{1}$. We assume that $\beta>0$ so that the probability of DLT is an increasing function of dose. The MTD, $\gamma$, is defined as the dose that is expected to produce DLT in a specified proportion $\theta$ of patients:

$$
P(Y=2 \mid x=\gamma)=F\left(\alpha_{2}+\beta \gamma\right)=\theta
$$

The value chosen for the target probability $\theta$ depends on the nature and clinical manageability of the DLT; it is set relatively high when the DLT is a transient, correctable, or nonfatal condition and low when it is lethal or life threatening. Suppose that dose levels in the trial are selected in the interval $\left[X_{\min }, X_{\max }\right]$.

\subsubsection{Likelihood}

Let $D_{n}=\left\{\left(x_{i}, Y_{i}\right), i=1, \ldots, n\right\}$ be the data after enrolling $n$ patients to the trial. The likelihood function for the parameters $\alpha_{1}, \alpha_{2}$, and $\beta$ is

$$
\begin{aligned}
L\left(\alpha_{1}, \alpha_{2}, \beta \mid D_{n}\right)= & \prod_{i=1}^{n}\left[1-F\left(\alpha_{1}+\beta x_{i}\right)\right]^{I\left(Y_{i}=0\right)}\left[F\left(\alpha_{1}+\beta x_{i}\right)-F\left(\alpha_{2}+\beta x_{i}\right)\right]^{I\left(Y_{i}=1\right)} \\
& \times\left[F\left(\alpha_{2}+\beta x_{i}\right)\right]^{I\left(Y_{i}=2\right)},
\end{aligned}
$$

where $I(\cdot)$ is the indicator function. 
We reparameterize model (2.2) in terms of $\rho_{0}=P\left(Y=2 \mid x=X_{\min }\right)$, the probability that a DLT manifests within the first cycle of therapy for a patient given dose $x=X_{\min }, \rho_{1}=$ $P\left(Y \geq 1 \mid x=X_{\min }\right)$, the probability that a grade 2 or more toxicity manifests within the first cycle of therapy for a patient given dose $x=X_{\min }$, and the MTD $\gamma$. This reparameterization is convenient to clinicians since $\gamma$ is the parameter of interest. Assuming that the dose is standardized to be in the interval $[0,1]$, it can be shown that

$$
\begin{gathered}
\alpha_{1}=F^{-1}\left(\rho_{1}\right), \quad \alpha_{2}=F^{-1}\left(\rho_{0}\right), \\
\beta=\frac{1}{r}\left(F^{-1}(\theta)-F^{-1}\left(\rho_{0}\right)\right) .
\end{gathered}
$$

The conditions $\alpha_{2} \leq \alpha_{1}, \beta>0$, and (2.2) imply that $0 \leq \rho_{0} \leq \rho_{1}$ and $0 \leq \rho_{0} \leq \theta$. Define

$$
\begin{aligned}
& F_{1}\left(\rho_{0}, \rho_{1}, \gamma ; x\right)=F\left(F^{-1}\left(\rho_{1}\right)+\left(F^{-1}(\theta)-F^{-1}\left(\rho_{0}\right)\right) \frac{x}{\gamma}\right) \\
& F_{2}\left(\rho_{0}, \rho_{1}, \gamma ; x\right)=F\left(F^{-1}\left(\rho_{0}\right)+\left(F^{-1}(\theta)-F^{-1}\left(\rho_{0}\right)\right) \frac{x}{\gamma}\right) .
\end{aligned}
$$

Using (2.4), (2.5), and (2.6), the likelihood of the reparameterized model is

$$
\begin{aligned}
L\left(\rho_{0}, \rho_{1}, \gamma \mid D_{n}\right)= & \prod_{i=1}^{n}\left[1-F_{1}\left(\rho_{0}, \rho_{1}, \gamma ; x_{i}\right)\right]^{I\left(Y_{i}=0\right)}\left[F_{1}\left(\rho_{0}, \rho_{1}, \gamma ; x_{i}\right)-F_{2}\left(\rho_{0}, \rho_{1}, \gamma ; x_{i}\right)\right]^{I\left(Y_{i}=1\right)} \\
& \times\left[F_{2}\left(\rho_{0}, \rho_{1}, \gamma ; x_{i}\right)\right]^{I\left(Y_{i}=2\right)} .
\end{aligned}
$$

\subsubsection{Prior and Posterior Distributions}

Let $g\left(\rho_{0}, \rho_{1}, \gamma\right)$ be the prior distribution on $\Omega$, where $\Omega=\{(x, y, z): 0 \leq x \leq \theta, x \leq y \leq$ $\left.1, X_{\min } \leq z \leq X_{\max }\right\}$. Using Bayes rule, the posterior distribution of the model parameters is proportional to the product of the likelihood and prior distribution

$$
\pi\left(\rho_{0}, \rho_{1}, y \mid D_{n}\right) \propto L\left(\rho_{0}, \rho_{1}, \gamma \mid D_{n}\right) \times g\left(\rho_{0}, \rho_{1}, \gamma\right)
$$

We designed an MCMC sampler based on the Metropolis-Hastings algorithm $[47,48]$ to obtain model operating characteristics. We also used WinBUGS [49] to estimate features of the posterior distribution of the MTD and design a trial. The WinBUGS code is included in the Appendix section. In the absence of prior information about the MTD and probability of DLT at $X_{\min }$, we specify vague priors for the model parameters as follows:

$$
\begin{gathered}
\gamma \sim \operatorname{Unif}\left[X_{\min }, X_{\max }\right] \\
\rho_{0} \sim \operatorname{Unif}[0, \theta] \\
\rho_{1} \mid \rho_{0} \sim \operatorname{Unif}\left[\rho_{0}, 1\right] .
\end{gathered}
$$




\subsubsection{Trial Design}

Dose levels in the trial are selected in the interval $\left[X_{\min }, X_{\max }\right]$. The adaptive design proceeds as follows. The first patient receives a dose $x_{1}>X_{\min }$ that is deemed to be safe by the clinician. Denote by $\Pi k(\gamma)=\Pi\left(\gamma \mid D_{k}\right)$ the marginal posterior cdf of the MTD, $k=1, \ldots, n-1$. The $(k+1)$-st patient receives the dose $x_{k+1}=\Pi_{k}^{-1}(\alpha)$ so that the posterior probability of exceeding the MTD is equal to the feasibility bound $\alpha$. This is the overdose protection property of EWOC, where at each stage of the design, we seek a dose to allocate to the next patient while controlling the posterior probability of exposing patients to toxic dose levels. The trial proceeds until a predetermined number of $n$ patients are enrolled to the trial. At the end of the trial, we estimate the MTD as $\hat{\gamma}=\Pi_{n}^{-1}(\alpha)$.

\section{Properties of EWOC-POM}

\subsection{Characteristics of EWOC-POM}

The proposed design EWOC-POM assigns dose levels to future patients by taking into account the maximum observed toxicity grade during the first cycle of therapy according to the following properties.

(i) At each stage of the design, we seek a dose to allocate to the next patient while controlling the posterior probability of exposing patients to toxic dose levels.

(ii) If the maximum grade of toxicity experienced by patient $k-1$ within one cycle of therapy is grade 2 , then the dose allocated to patient $k$ is lower than the dose that would have been given to patient $k$ had the maximum grade of toxicity experienced by patient $k-1$ been grade 0 or 1 .

Property (i) is the overdose protection defining characteristic of EWOC which is also satisfied by EWOC-POM. Property (ii) is naturally appealing because when a patient experiences grade 2 dose-related toxicity, then it is ethical not to increase the dose by the same amount had this patient exhibited grade 0 or 1 toxicity at the most. Characteristic (ii) is summarized in the following theorem.

Theorem 3.1. Let $D_{k}=\left\{\left(Y_{1}, x_{1}\right), \ldots,\left(Y_{k}, x_{k}\right)\right\}$ be the data on the first $k$ patients generated by the design described in Section 2.1.3, and, $\Pi_{k}\left(\gamma ; Y_{k}\right)$ be the cdf of $\gamma$ given the data $D_{k}$. Let $x_{k+1}=$ $\Pi_{k}^{-1}\left(\alpha ; Y_{k}\right)$ and $x_{k+1}^{\prime}=\Pi_{k}^{-1}\left(\alpha ; Y_{k}^{\prime}\right)$. Suppose that for all $x \in\left[X_{\min }, X_{\max }\right]$ and all $\left(\rho_{0}, \rho_{1}\right)$ such that $0 \leq$ $\rho_{0} \leq \rho_{1} \leq 1$ and $\rho_{0} \leq \theta,\left(F_{1}-F_{2}\right) /\left(1-F_{1}\right)$ is a monotonically decreasing function in $\gamma$. Then, $x^{\prime}{ }_{k+1} \geq x_{k+1}$ whenever $Y_{k}{ }^{\prime}=0$ and $Y_{k}=1$.

The proof of Theorem 3.1 is given in the Appendix section. It is easy to verify that the monotonicity condition of Theorem 3.1 holds for the logistic function $F(w)=1 /\left(1+e^{-w}\right)$. Using this link function and the uniform priors given in (2.9) with $\theta=0.33$, Figure 1 gives all possible dose assignments for patients 1 and 2 and selected situations for patients 3 and 4 using the trial design described in Section 2.1.3. The dose has been standardized so that $X_{\min }=0$ and $X_{\max }=1$, and the first patient is given dose 0.10 . If this patient experiences grade 0 or 1 toxicity at the most, the dose recommended for patient 2 is 0.36 . On the other hand, if patient 1 experiences grade 2 toxicity at the most, the dose recommended for patient 2 is 0.22 , much lower than 0.36 . This figure also illustrates some of the coherence properties stated in Theorem 3.2 below. 


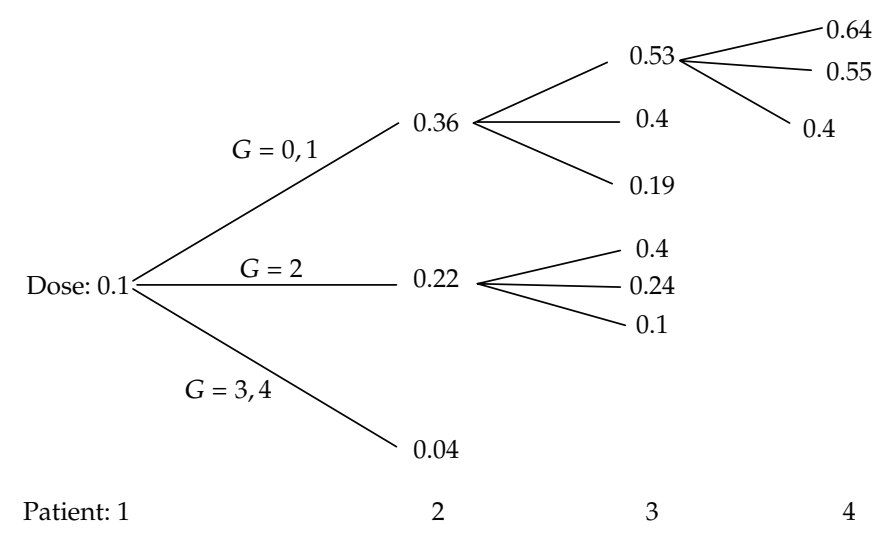

Figure 1: Tree of possible dose allocations for patients 1 and 2 and selected situations for patients 3 and 4 . $G=0,1$ corresponds to $Y=0, G=2$ corresponds to $Y=1$, and $G=3,4$ corresponds to $Y=2$ or DLT.

\subsection{Coherence of EWOC-POM}

In cancer phase I clinical trials, it is ethical not to increase a dose of a cytotoxic agent for the next patient if the previously treated patient exhibited DLT when given the same dose level. Furthermore, it is desirable not to decrease the dose of an agent for the next patient if the previously treated patient did not experience DLT when given that same dose level. These two properties are known as coherence in escalation and de-escalation, respectively, see Cheung [50]. A design that satisfies both of these properties is said to be coherent. Tighiouart and Rogatko [23] show that EWOC is coherent. The next theorem states some of the coherence properties of EWOC-POM.

Theorem 3.2. Suppose that for all $x \in\left[X_{\min }, X_{\max }\right]$ and all $\left(\rho_{0}, \rho_{1}\right)$ such that $0 \leq \rho_{0} \leq \rho_{1} \leq 1$ and $\rho_{0} \leq \theta, F_{1}$ and $F_{2}$ are monotonically decreasing in $\gamma$. Then the design EWOC-POM described in 2.1.3 is coherent in deescalation. Furthermore, if the toxicity response for patient $k$ is $Y_{k}=0$, then the dose allocated to patient $k+1$ satisfies $x_{k+1} \geq x_{k}$.

The proof of Theorem 3.2 is given in the Appendix section.

\section{Simulation Studies}

We compare the design operating characteristics of EWOC-POM with the original EWOC by simulating a large number of trials under several scenarios. We used the logistic function $F(w)=1 /\left(1+e^{-w}\right)$ to model the dose-toxicities relationship in (2.2). EWOC was implemented as in Tighiouart et al. [22] using the same logistic function to model the dose-toxicity relationship. For all scenarios, we standardize the dose to be in the interval $[0,1], \theta=0.33$, the feasibility bound $\alpha=0.25$, and the trial sample size is $n=30$. The priors in (2.9) were adopted for EWOC-POM. We also carried out design operating characteristics for $\theta=0.20$, and the conclusions regarding the performance of EWOC-POM relative to EWOC were essentially the same.

\subsection{Algorithm}

For a given scenario determined by $\rho_{0}, \rho_{1}$, and $\gamma$, the first patient receives dose 0 , and the next dose $x_{2}$ is determined according to the trial design described in 2.1.3. The second 


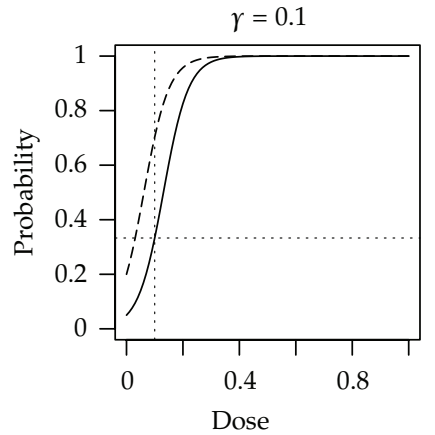

(a)

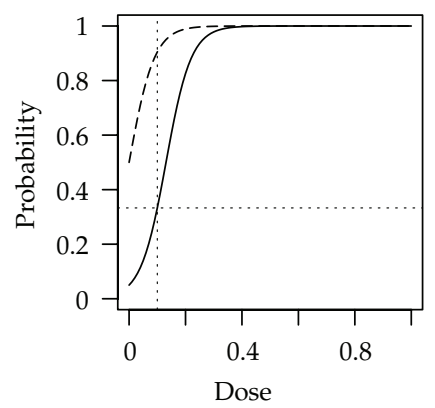

(d)

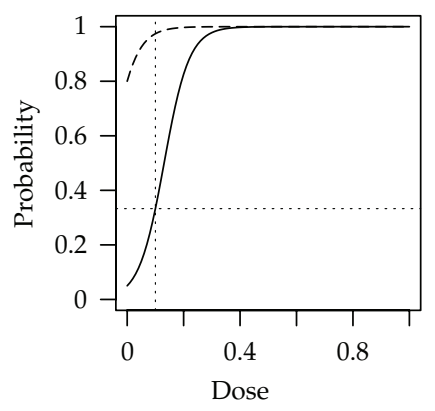

(g)

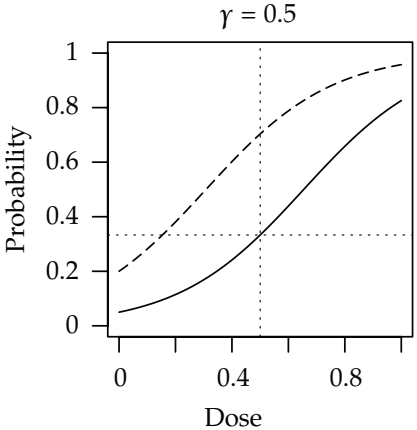

(b)

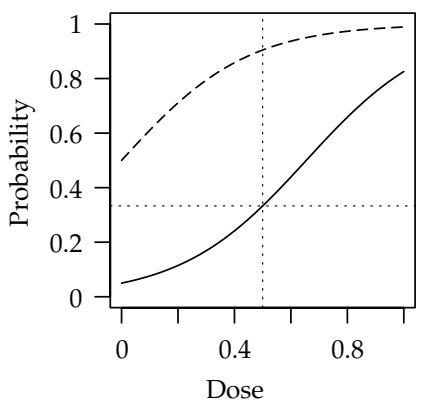

(e)

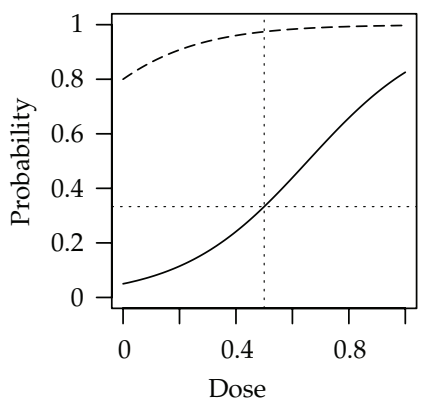

(h)

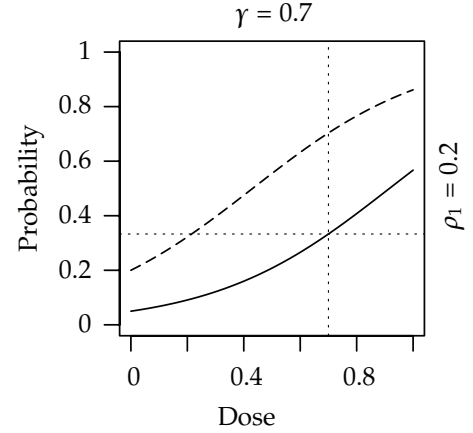

(c)

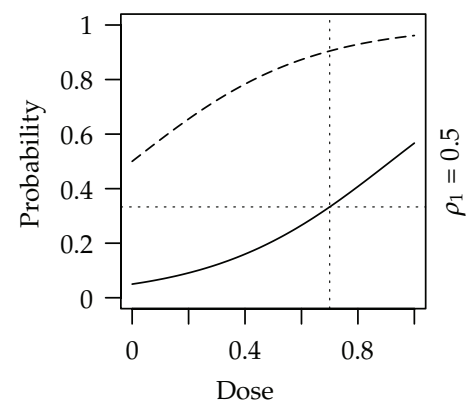

(f)

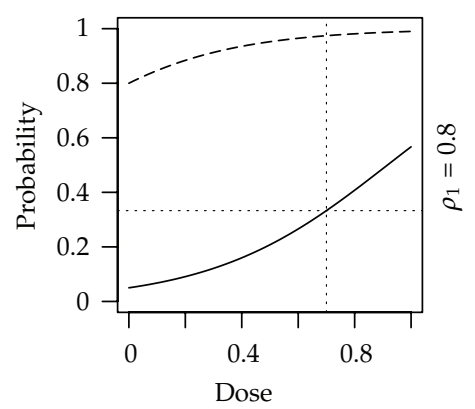

(i)

Figure 2: Dose-toxicity relationship for the nine scenarios considered in the design operations characteristics. The solid curves correspond to $P(Y=2 \mid x)=P(D L T \mid x)$ and the dashed curves in bold correspond to $P(Y>1 \mid x)$. The horizontal dashed lines represent the target probability of DLT $\theta=0.33$ and the vertical lines correspond to the true values of the MTD $\gamma$.

response $y_{2}$ is then generated from model (2.2) reparameterized in terms of $\rho_{0}, \rho_{1}$, and $\gamma$ with $x=x_{2}$. This process is repeated until all $n$ patients have been enrolled to the trial. We considered 9 scenarios corresponding to a fixed value for $\rho_{0}=0.05$, three values of $\rho_{1}, 0.2$, 0.5 , and 0.8 , and three values of the MTD $\gamma, 0.1,0.5$, and 0.7 . The corresponding dose-toxicity relationships for these nine scenarios are illustrated in Figure 2. For each model and each scenario, we simulated $M=1000$ trials. EWOC and EWOC-POM were compared in terms of the proportion of patients exhibiting DLT, the average bias, bias ave $=M^{-1}\left(\sum_{i=1}^{M} \widehat{\gamma}_{i}-\gamma_{\text {true }}\right)$, and the estimated mean square error MSE $=M^{-1}\left(\sum_{i=1}^{M}\left(\widehat{\gamma}_{i}-\gamma_{\text {true }}\right)^{2}\right)$, where $\widehat{\gamma}_{i}$ is the Bayes 


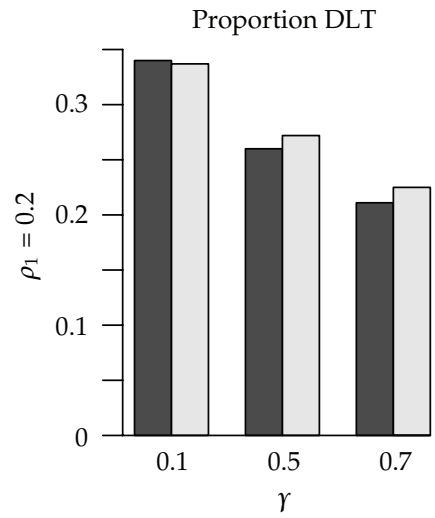

(a)

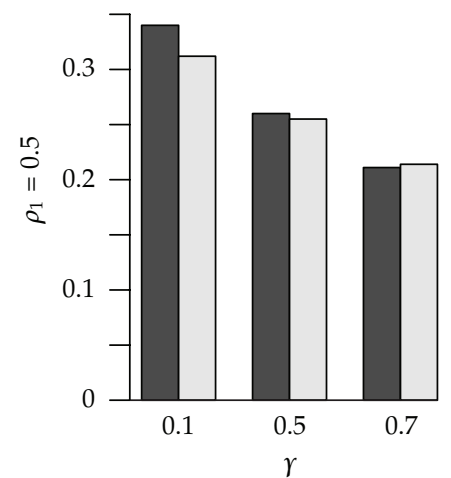

(d)

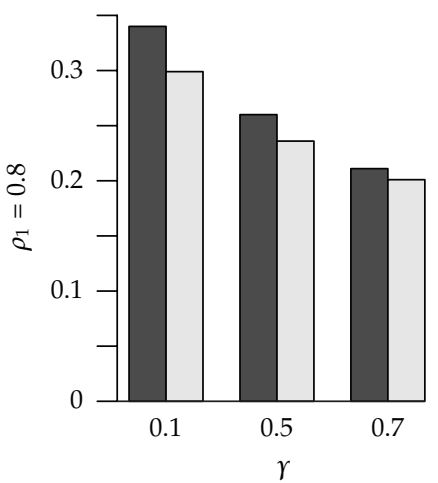

EWOC

$\square$ EWOC-POM

(g)

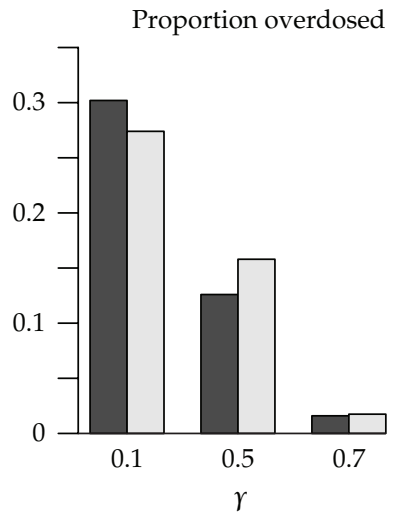

(b)

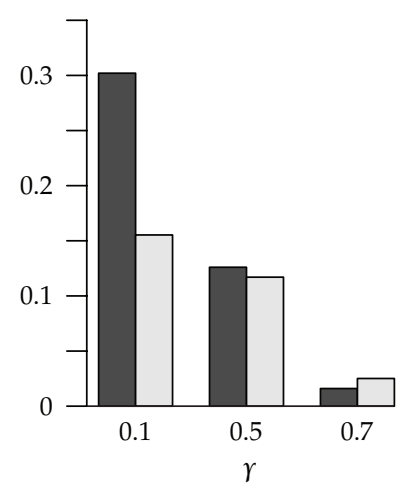

(e)

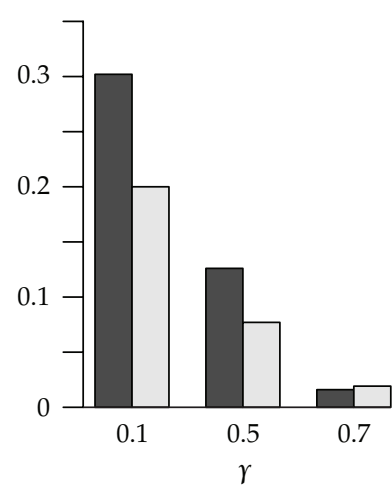

EWOC

$\square$ EWOC-POM

(h)

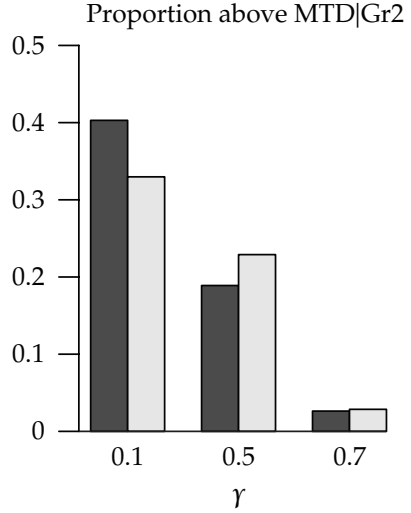

(c)

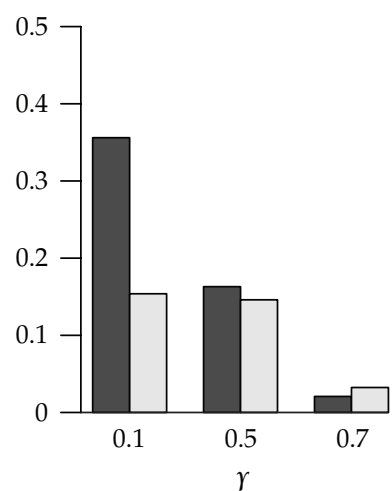

(f)

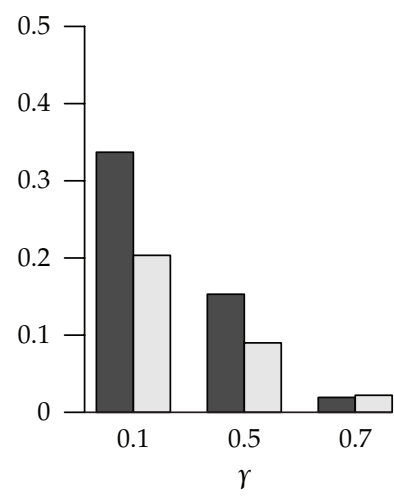

- EWOC

$\square$ EWOC-POM

(i)

Figure 3: Summary statistics for trial safety for EWOC and EWOC-POM under all scenarios. Each graph represents mean proportion obtained from all patients from all 1000 simulated trials. 
Table 1: Percent of trials with estimated MTD within 5\% of the dose range and $10 \%$ of the dose range of the true MTD $\gamma$ and percent of trials for which the rate of DLT exceeds $40 \%$ for EWOC and EWOC-POM under the nine scenarios.

\begin{tabular}{|c|c|c|c|c|c|c|}
\hline \multirow{3}{*}{ Scenarios } & \multicolumn{4}{|c|}{ Percent of trial with estimated MTD } & \multirow{2}{*}{\multicolumn{2}{|c|}{$\begin{array}{c}\text { Percent of Trial } \\
\text { with rate of DLT }>0.4\end{array}$}} \\
\hline & \multicolumn{2}{|c|}{ Within 0.05 of $\gamma$} & \multicolumn{2}{|c|}{ Within 0.10 of $\gamma$} & & \\
\hline & EWOC & EWOC-POM & EWOC & EWOC-POM & EWOC & EWOC-POM \\
\hline$\gamma=0.1, \rho_{1}=0.2$ & 98.3 & 98.4 & 100 & 100 & 7.5 & 6.6 \\
\hline$\gamma=0.1, \rho_{1}=0.5$ & 98.3 & 97.5 & 100 & 100 & 7.5 & 3.0 \\
\hline$\gamma=0.1, \rho_{1}=0.8$ & 98.3 & 96.4 & 100 & 100 & 7.5 & 2.9 \\
\hline$\gamma=0.5, \rho_{1}=0.2$ & 39.6 & 40.5 & 70.3 & 71.3 & 0.2 & 0.0 \\
\hline$\gamma=0.5, \rho_{1}=0.5$ & 39.6 & 35.6 & 70.3 & 63.2 & 0.2 & 0.0 \\
\hline$\gamma=0.5, \rho_{1}=0.8$ & 39.6 & 31.0 & 70.3 & 59.4 & 0.2 & 0.0 \\
\hline$\gamma=0.7, \rho_{1}=0.2$ & 24.3 & 27.6 & 49.1 & 53.3 & 0.0 & 0.0 \\
\hline$\gamma=0.7, \rho_{1}=0.5$ & 24.3 & 23.2 & 49.1 & 45.7 & 0.0 & 0.0 \\
\hline$\gamma=0.7, \rho_{1}=0.8$ & 24.3 & 20.1 & 49.1 & 37.1 & 0.0 & 0.0 \\
\hline
\end{tabular}

estimate of the posterior distribution of the MTD at the end of the $i$ th trial with respect to the asymmetric loss function:

$$
L(x, \gamma)= \begin{cases}\alpha(\gamma-x) & \text { if } x \leq \gamma \\ (1-\alpha)(x-\gamma) & \text { if } x>\gamma\end{cases}
$$

We also compared the models with respect to the proportion of patients that were overdosed. Here, a dose $x$ is defined as an overdose if $x>x^{*}$, where $x^{*}$ is defined as the dose for which $P\left(\right.$ DLT $\left.\mid x^{*}\right)=\theta+0.05$. This probability is calculated using the parameter values from the corresponding scenario. These models are further compared with respect to the proportion of patients that were overdosed given that the previously treated patient exhibited grade 2 toxicity. Finally, we compared EWOC-POM to EWOC in terms of the proportion of trials for which the probability of DLT exceeds 0.4 . This gives us an estimate of the probability that a prospective trial will result in an excessively high DLT rate. As for the proportion of trials with correct MTD recommendation, we presented percent of trials with estimated MTD within $10 \%$ and $20 \%$ of the dose range of the true MTD for EWOC-POM and EWOC.

\subsection{Results}

Figure 3 shows that the proportion of patients exhibiting DLT is always less than $34 \%$ for both EWOC and EWOC-POM under all scenarios and $4 \%$ fewer patients experiencing DLT under EWOC-POM when the MTD is small $(\gamma=0.1)$ and $\rho_{1}=0.8$. The same figure shows that the proportion of patients that are overdosed using EWOC is uniformly higher relative to EWOC-POM when the MTD is small. The same trend is observed when $\gamma=0.5$ except when $\rho_{1}=0.2$. The difference in the proportion of patients being overdosed when $\gamma=0.7$ is negligible. The last panel of Figure 3 shows that the proportion of patients that are overdosed given that the previously treated patient exhibited grade 2 toxicity using EWOC is uniformly higher relative to EWOC-POM when $\gamma=0.1,0.5$ except when $\rho_{1}=0.2$. The difference in these proportions when $\gamma=0.7$ is negligible. The last two columns of Table 1 show that the percent of trials with DLT rate of 0.4 or more is $7.5 \%$ at the most for EWOC and $6.6 \%$ for EWOCPOM. A more detailed comparison is shown in Figure 4, where side-by-side box plots of 


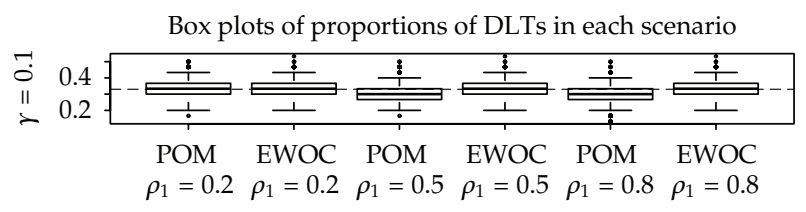

(a)

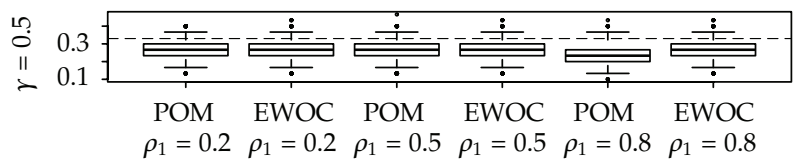

(b)

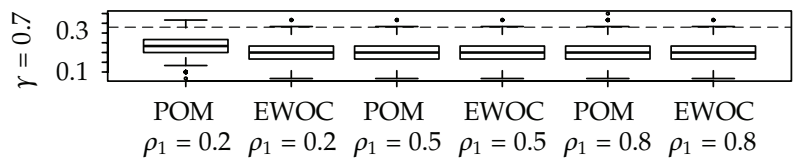

(c)

Figure 4: Box plots for the proportion of DLTs for EWOC-POM and EWOC under the nine scenarios. Each box plot was constructed from the DLT rates of the 1000 simulated trials. The dashed horizontal line corresponds to the target probability of DLT $\theta=0.33$.

the distributions of the proportion of DLTs for EWOC-POM and EWOC under the nine scenarios are displayed. These results show that EWOC-POM maintains the safety of the trial relative to EWOC and is much safer when the true MTD is close to the minimum dose by reducing the number of patients that are exposed to toxic doses.

Figure 5 shows that the estimated MTDs using EWOC and EWOC-POM are very close in general, with the highest difference observed when $\rho_{1}=0.8$. This is reflected by the estimated bias and RMSE shown in Figure 5. This is expected since EWOC-POM is characterized by a conservative dose escalation when a patient experiences grade 2 toxicity. The highest absolute value of the bias is 0.04 and is achieved when $\gamma=0.5,0.7$ and $\rho_{1}=0.8$. This constitutes $4 \%$ of the range of the dose and is practically not significant. The percent of trials with estimated MTD within 5\% of the dose range and 10\% of the dose range of the true MTD $\gamma$ under the nine scenarios are shown in columns $2-5$ of Table 1 . These results further confirm that the precision of the estimate of the MTD is similar between the two models, with a higher precision for EWOC achieved when $\gamma=0.5$ and $\rho_{1}=0.8$. Values other than $5 \%$ and $10 \%$ of the dose range were also used, and the conclusions were essentially the same. We conclude that EWOC-POM maintains the efficiency of the trial relative to EWOC for all practical purpose.

These simulation results suggest that EWOC-POM is a good alternative design for cancer phase I clinical trials which takes into account the ethical consideration that dose escalation in the absence of DLT is mitigated by the occurrence of a grade 2 toxicity.

\subsection{Model Robustness}

Model robustness with respect to assumption of proportional odds model in (2.2) was assessed by simulating the toxicity responses from a nonproportional odds model:

$$
P(Y \geq j \mid x)=F\left(\alpha_{j}+\beta_{j} x\right), \quad j=1,2
$$




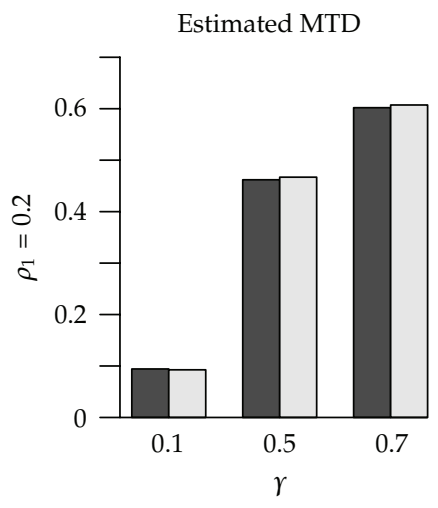

(a)

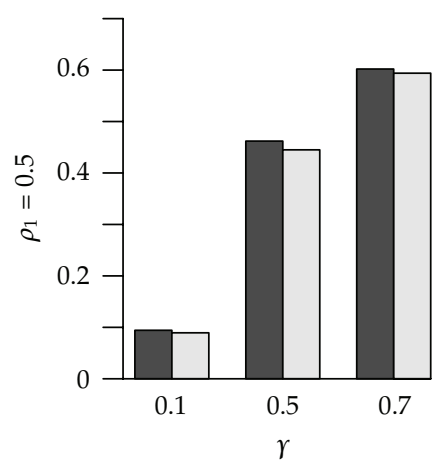

(d)

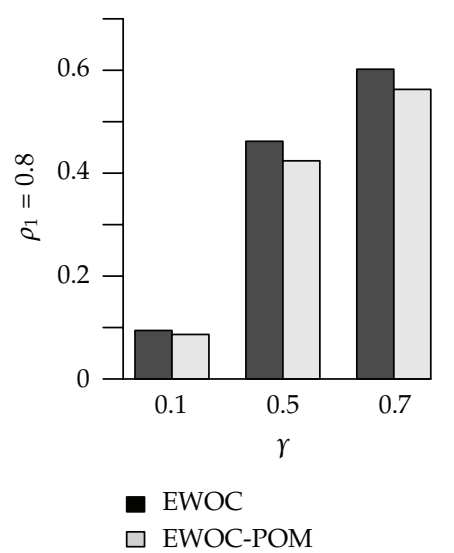

(g)

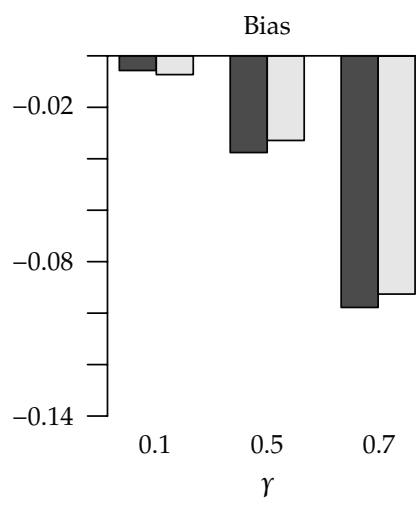

(b)

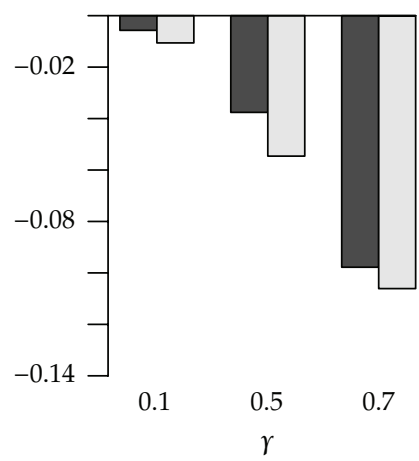

(e)

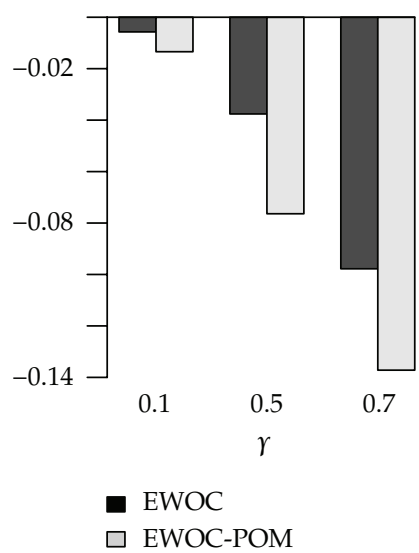

(h)

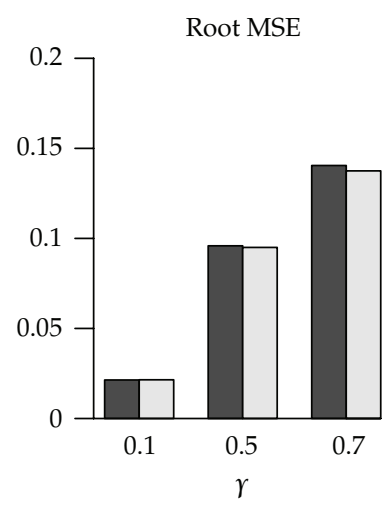

(c)

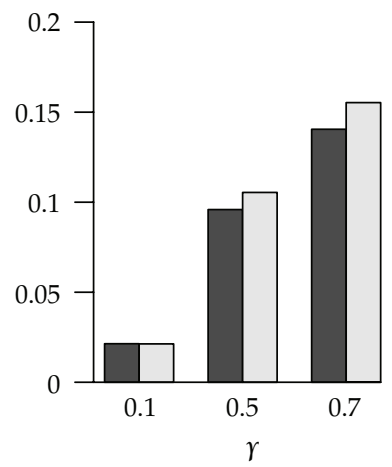

(f)

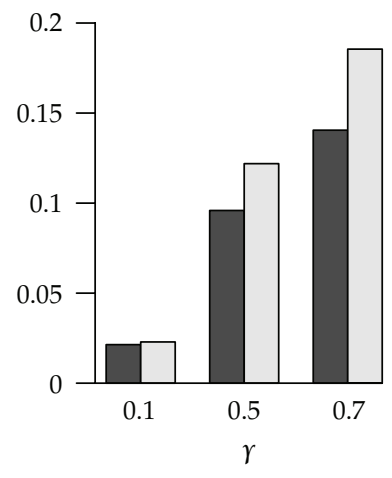

- EWOC

$\square$ EWOC-POM

(i)

Figure 5: Summary statistics for trial efficiency for EWOC and EWOC-POM under all scenarios. Each graph represents a mean value obtained from all patients from all 1000 trials.

The same logistic link function $F(w)=1 /\left(1+e^{-w}\right)$ was used. We considered two different models $\mathrm{M}_{1}$ and $\mathrm{M}_{2}$ corresponding to the set of parameters $\alpha_{2}=-3.94, \beta_{1}=22.36, \beta_{2}=$ 32.36 for model $\mathrm{M}_{1}$ and $\alpha_{2}=-1.94, \beta_{1}=22.36, \beta_{2}=12.36$ for $\mathrm{M}_{2}$. For each model $M_{i}, i=1,2$, we considered three different values for the intercept term $\alpha_{1}, \alpha_{1}=-1.38,0.00,1.38$ which correspond to $\rho_{1}=0.2,0.5,0.8$. These parameters have been selected so that $\rho_{0}=0.020$ for 


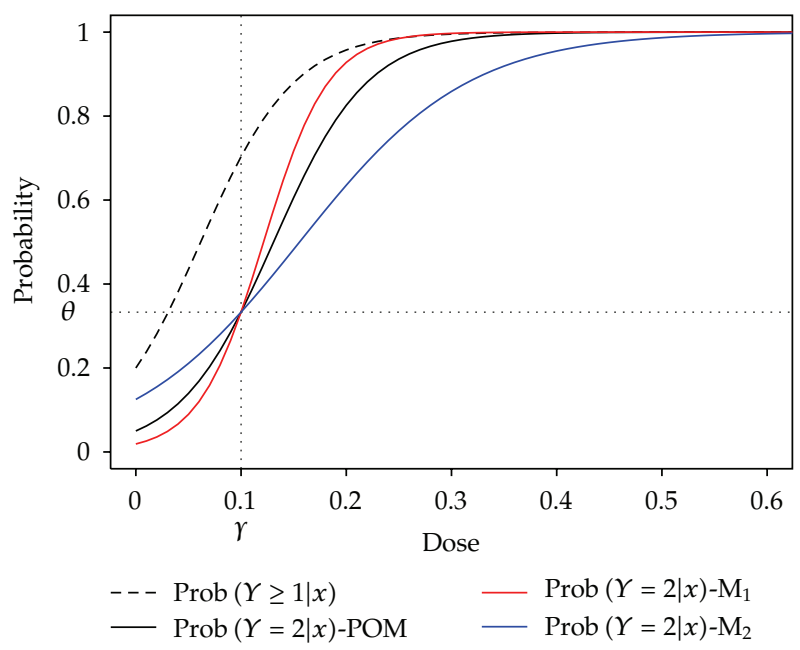

Figure 6: Dose-toxicity relationship under the proportional (EWOC-POM) and nonproportional odds models $\left(\mathrm{M}_{1}\right.$ and $\left.\mathrm{M}_{2}\right)$ when the true MTD $\gamma=0.1$. The dashed horizontal line corresponds to the target probability of DLT $\theta=0.33$.

model $\mathrm{M}_{1}, \rho_{0}=0.126$ for model $\mathrm{M}_{2}$, and the true MTD is $\gamma=0.1$. Figure 6 shows the graphs of the probabilities of DLT and probability of grade 2 or more toxicity as a function of dose for the proportional odds model POM and models $\mathrm{M}_{1}$ and $\mathrm{M}_{2}$ when $\rho_{1}=0.2$. For each scenario, we simulated 1000 trials with $n=30$ patients where at each stage of the trial, the next dose is calculated using the trial design described in 2.1 .3 as in Section 4.1 but the toxicity response is generated using the nonproportional odds model (4.2). The simulation results are summarized in Table 2. The maximum difference in proportion of patients exhibiting DLT (averaged across the simulated trials) between model $M_{i}, i=1,2$ and EWOC-POM is 3\%. Under model $\mathrm{M}_{2}$, the proportion of patients that are overdosed is higher than EWOC-POM, and this proportion is $13 \%$ higher when $\rho_{1}=0.2$.

The percent of trials with DLT rate exceeding 0.4 is less than $15 \%$ in all cases. This percent is highest for model $\mathrm{M}_{2}$; however, this is still relatively small compared to the results obtained in [42]. The percent of trials with estimated MTD within $10 \%$ of the dose range of the true MTD is $100 \%$ between the three models and across all scenarios and very good within $5 \%$ of the dose range of the true MTD.

In summary, EWOC-POM seems to be robust to model misspecification when the true MTD is near the initial dose. On the other hand, the model is sensitive to model misspecification when the true MTD is high but the safety of the trial is not compromised. We also conducted similar simulations (results not shown) when the true MTD is $\gamma=0.5,0.7$. We found that under all scenarios, the proportion of patients exhibiting DLT is always less than $33 \%$ but the bias tends to be higher for high values of $\rho_{1}$ and $\gamma$. This is the case when the probability of DLT curve increases very slowly as a function of dose which results in a very slow dose escalation scheme.

\section{Discussion}

In this paper, we proposed a Bayesian adaptive design for dose-finding studies in cancer phase I clinical trials. The method addresses the ethical concern regarding dose escalation in 
Table 2: Summary statistics for trial safety and efficiency under model misspecification when the true MTD $r=0.1$. Rows $2-6$ give the summary statistics based on all patients from all 1000 trials.

\begin{tabular}{|c|c|c|c|c|}
\hline \multirow{2}{*}{ Statistics } & \multirow{2}{*}{ Model } & \multicolumn{3}{|c|}{$\rho_{1}$} \\
\hline & & 0.2 & 0.5 & 0.8 \\
\hline \multirow{3}{*}{ Proportion of DLTs } & EWOC-POM & 0.337 & 0.312 & 0.299 \\
\hline & $\mathrm{M}_{1}$ & 0.328 & 0.304 & 0.289 \\
\hline & $\mathrm{M}_{2}$ & 0.357 & 0.342 & 0.331 \\
\hline \multirow{3}{*}{ Proportion overdosed } & EWOC-POM & 0.274 & 0.155 & 0.200 \\
\hline & $\mathrm{M}_{1}$ & 0.240 & 0.203 & 0.173 \\
\hline & $\mathrm{M}_{2}$ & 0.404 & 0.351 & 0.322 \\
\hline \multirow{3}{*}{ MTD estimate } & EWOC-POM & 0.093 & 0.090 & 0.087 \\
\hline & $\mathrm{M}_{1}$ & 0.094 & 0.092 & 0.090 \\
\hline & $\mathrm{M}_{2}$ & 0.095 & 0.090 & 0.086 \\
\hline \multirow{3}{*}{ Bias } & EWOC-POM & -0.007 & -0.010 & -0.013 \\
\hline & $\mathrm{M}_{1}$ & -0.006 & -0.007 & -0.010 \\
\hline & $\mathrm{M}_{2}$ & -0.005 & -0.010 & -0.013 \\
\hline \multirow{3}{*}{ Root MSE } & EWOC-POM & 0.022 & 0.021 & 0.023 \\
\hline & $\mathrm{M}_{1}$ & 0.016 & 0.016 & 0.017 \\
\hline & $\mathrm{M}_{2}$ & 0.027 & 0.029 & 0.031 \\
\hline \multirow{3}{*}{ Percent of trial with DLT rate $>40 \%$} & EWOC-POM & 6.6 & 3.0 & 2.9 \\
\hline & $\mathrm{M}_{1}$ & 1.5 & 1.2 & 0.8 \\
\hline & $\mathrm{M}_{2}$ & 14.7 & 11.6 & 12.6 \\
\hline \multirow{3}{*}{ Percent of trial with estimated MTD within 0.05 of $\gamma$} & EWOC-POM & 98.4 & 97.5 & 96.4 \\
\hline & $\mathrm{M}_{1}$ & 99.7 & 99.5 & 99.5 \\
\hline & $\mathrm{M}_{2}$ & 91.1 & 91.2 & 88.1 \\
\hline \multirow{3}{*}{ Percent of trial with estimated MTD within 0.10 of $\gamma$} & EWOC-POM & 100.0 & 100.0 & 100.0 \\
\hline & $\mathrm{M}_{1}$ & 100.0 & 100.0 & 100.0 \\
\hline & $\mathrm{M}_{2}$ & 100.0 & 100.0 & 100.0 \\
\hline
\end{tabular}

the absence of DLT. Specifically, if the current patient experiences drug-related grade 2 toxicity at the most, then it is ethical not to escalate the dose for the next patient by the same amount as the one had the current patient experienced a maximum of grade 0 or 1 toxicity. The method termed EWOC-POM is an extension of EWOC by accommodating an intermediate grade 2 toxicity to the model. We used a proportional odds model to describe the dosetoxicities relationship for simplicity. We proved that if the maximum grade of toxicity experienced by patient $k-1$ within one cycle of therapy is grade 2 , then the dose allocated to patient $k$ is lower than the dose that would have been given to patient $k$ had the maximum grade of toxicity experienced by patient $k-1$ been grade 0 or 1 . Furthermore, we also showed that the coherence properties of EWOC are maintained.

We studied design operating characteristics by simulating a large number of trials under different scenarios of the dose-toxicity relationships. EWOC-POM was compared to EWOC with respect to the primary goals of cancer phase I trials, safety and efficiency of the estimate of the MTD. We found that in general, the safety of the trial is not compromised when we account for an intermediate grade 2 toxicity. In particular, when the unknown MTD is close to the initial dose, a substantial number of patients are overdosed when using EWOC relative to EWOC-POM, and if the current patient experiences grade 2 toxicity, then the next patient is more likely to be overdosed using EWOC compared to EWOC-POM. The loss in efficiency of the estimate of the MTD by introducing an extra parameter to the model is very 
marginal as was shown by the simulation results of the various scenarios. We also showed that the proportional odds assumption is robust to model misspecification when the true MTD is close to the minimum dose. However, the bias of the estimate of the MTD increases as a function of the MTD under model misspecification. In any case, safety of the trial as assessed by the number of patients exhibiting DLT and number of patients that are overdosed was never compromised.

We have shown that Theorem 3.1 holds under the proportional odds assumption using EWOC scheme with link functions satisfying monotonicity conditions as a function of the MTD. One can easily prove that a similar ethical constraint stated in Theorem 3.1 can be established using the Bayesian CRM originally proposed in O'Quigley et al. [3]. In fact, a more general theorem can be established for a Bayes estimate using a general convex loss function. Extensions of EWOC-POM to accommodate patients' baseline characteristics ([30]) and time to event DLT for late onset toxicities are being investigated. In conclusion, EWOC-POM is a good alternative design to EWOC if clinicians expect to see a substantial number of grade 2 toxicities induced by the agent or drug combinations under study.

\section{Appendix}

Proof of Theorem 3.1. To simplify notation and presentation of the proof, we assume that $X_{\min }=0, \rho_{0} \leq \rho_{1}$ are fixed, and let us drop the nuisance parameters $\rho_{0}$ and $\rho_{1}$ from $F_{1}\left(\rho_{0}, \rho_{1}\right.$, $\gamma ; x)$, and $F_{2}\left(\rho_{0}, \rho_{1}, \gamma ; x\right)$. Let $L_{k}(\gamma)=L_{k}\left(\rho_{0}, \rho_{1}, \gamma \mid D_{k}\right)$, and $\pi(\gamma)$ be a proper prior density for $\gamma$. If $Y_{k}=0, L_{k}(\gamma)=L_{k-1}(\gamma)\left[1-F_{1}\left(\gamma ; x_{k}\right)\right]$, and if $Y_{k}=1, L_{k}(\gamma)=L_{k-1}(\gamma)\left[F_{1}\left(\gamma ; x_{k}\right)-F_{2}\left(\gamma ; x_{k}\right)\right]$. Using Bayes' rule,

$$
\begin{aligned}
\prod_{k}(t ; 0)- & \prod_{k}(t ; 1) \\
= & \frac{\int_{0}^{t} L_{k-1}(\gamma)\left[1-F_{1}\left(\gamma ; x_{k}\right)\right] \pi(\gamma) d \gamma}{\int_{0}^{1} L_{k-1}(\gamma)\left[1-F_{1}\left(\gamma ; x_{k}\right)\right] \pi(\gamma) d \gamma}-\frac{\int_{0}^{t} L_{k-1}(\gamma)\left[F_{1}\left(\gamma ; x_{k}\right)-F_{2}\left(\gamma ; x_{k}\right)\right] \pi(\gamma) d \gamma}{\int_{0-1}^{1}(\gamma)\left[F_{1}\left(\gamma ; x_{k}\right)-F_{2}\left(\gamma ; x_{k}\right)\right] \pi(\gamma) d \gamma} \\
= & A^{-1}\left[\int_{0}^{t} \int_{0}^{1} L_{k-1}(\gamma) L_{k-1}\left(\gamma^{\prime}\right) \pi(\gamma) \pi\left(\gamma^{\prime}\right)\left[1-F_{1}\left(\gamma ; x_{k}\right)\right]\left[F_{1}\left(\gamma^{\prime} ; x_{k}\right)-F_{2}\left(\gamma^{\prime} ; x_{k}\right)\right] d \gamma^{\prime} d \gamma\right] \\
& -A^{-1}\left[\int_{0}^{t} \int_{0}^{1} L_{k-1}(\gamma) L_{k-1}\left(\gamma^{\prime}\right) \pi(\gamma) \pi\left(\gamma^{\prime}\right)\left[F_{1}\left(\gamma ; x_{k}\right)-F_{2}\left(\gamma ; x_{k}\right)\right]\left[1-F_{1}\left(\gamma^{\prime} ; x_{k}\right)\right] d \gamma^{\prime} d \gamma\right] \\
= & A^{-1}\left[\int_{0}^{t} \int_{t}^{1} L_{k-1}(\gamma) L_{k-1}\left(\gamma^{\prime}\right) \pi(\gamma) \pi\left(\gamma^{\prime}\right)\left[1-F_{1}\left(\gamma ; x_{k}\right)\right]\left[F_{1}\left(\gamma^{\prime} ; x_{k}\right)-F_{2}\left(\gamma^{\prime} ; x_{k}\right)\right] d \gamma^{\prime} d \gamma\right] \\
& -A^{-1}\left[\int_{0}^{t} \int_{t}^{1} L_{k-1}(\gamma) L_{k-1}\left(\gamma^{\prime}\right) \pi(\gamma) \pi\left(\gamma^{\prime}\right)\left[F_{1}\left(\gamma ; x_{k}\right)-F_{2}\left(\gamma ; x_{k}\right)\right]\left[1-F_{1}\left(\gamma^{\prime} ; x_{k}\right)\right] d \gamma^{\prime} d \gamma\right] \\
= & A^{-1}\left[\int _ { 0 } ^ { t } \int _ { t } ^ { 1 } L _ { k - 1 } ( \gamma ) L _ { k - 1 } ( \gamma ^ { \prime } ) \pi ( \gamma ) \pi ( \gamma ^ { \prime } ) \left\{\left[1-F_{1}\left(\gamma ; x_{k}\right)\right]\left[F_{1}\left(\gamma^{\prime} ; x_{k}\right)-F_{2}\left(\gamma^{\prime} ; x_{k}\right)\right]\right.\right. \\
& \left.\left.-\left[F_{1}\left(\gamma ; x_{k}\right)-F_{2}\left(\gamma ; x_{k}\right)\right]\left[1-F_{1}\left(\gamma^{\prime} ; x_{k}\right)\right]\right\} d \gamma^{\prime} d \gamma\right],
\end{aligned}
$$


where

$$
A=\iint_{0}^{1} L_{k-1}(\gamma) L_{k-1}\left(\gamma^{\prime}\right)\left[1-F_{1}\left(\gamma ; x_{k}\right)\right]\left[F_{1}\left(\gamma^{\prime} ; x_{k}\right)-F_{2}\left(\gamma^{\prime} ; x_{k}\right)\right] \pi(\gamma) \pi\left(\gamma^{\prime}\right) d \gamma^{\prime} d \gamma
$$

Since $\gamma \leq \gamma^{\prime}$ and $\left(F_{1}-F_{2}\right) /\left(1-F_{1}\right)$ is monotonically decreasing in $\gamma$, then $\left[F_{1}\left(\gamma^{\prime} ; x_{k}\right)-\right.$ $\left.F_{2}\left(\gamma^{\prime} ; x_{k}\right)\right] /\left[1-F_{1}\left(\gamma^{\prime} ; x_{k}\right)\right] \leq\left[F_{1}\left(\gamma ; x_{k}\right)-F_{2}\left(\gamma ; x_{k}\right)\right] /\left[1-F_{1}\left(\gamma ; x_{k}\right)\right]$, which implies that $[1-$ $\left.F_{1}\left(\gamma ; x_{k}\right)\right]\left[F_{1}\left(\gamma^{\prime} ; x_{k}\right)-F_{2}\left(\gamma^{\prime} ; x_{k}\right)\right] \leq\left[F_{1}\left(\gamma ; x_{k}\right)-F_{2}\left(\gamma ; x_{k}\right)\right]\left[1-F_{1}\left(\gamma^{\prime} ; x_{k}\right)\right]$. Hence, $\Pi_{k}(t ; 0) \leq$ $\Pi_{k}(t ; 1)$, which implies that $\Pi_{k}^{-1}(\alpha ; 0) \geq \Pi_{k}^{-1}(\alpha ; 1)$, that is, $x_{k+1} \geq x_{k+1}^{\prime}$. This completes the proof of Theorem 3.1.

Proof of Theorem 3.2. Using the notation in the proof of Theorem 3.1, if $Y_{k}=0$, then $L_{k}(\gamma)=$ $L_{k-1}(\gamma)\left[1-F_{1}\left(\gamma ; x_{k}\right)\right]$, and if $Y_{k}=2$, then $L_{k}(\gamma)=L_{k-1}(\gamma) F_{2}\left(\gamma ; x_{k}\right)$. Since both $F_{1}$ and $F_{2}$ are monotonically decreasing in $\gamma$, then the result of the theorem follows from the proof of Theorem 1 in Tighiouart and Rogatko [23].

\section{WinBUGS Code}

\# Assume that the toxicity response $Y$ has 3 categories:

$\# Y=1$ if patient has grade 0 or 1 toxicity

$\# Y=2$ if patient has a grade 2 toxicity

$\# Y=3$ if patient has grade 3 or 4 toxicity, that is, DLT

\# Dose is standardized between 0 and 1 .

model \{

for (i in $1: N)\{$

\# Likelihood

$\operatorname{logit}(\mathrm{eta}[\mathrm{i}, 1])<--(\operatorname{logit}(\mathrm{rho} 1))-(1 / \mathrm{mtd})^{*}(\operatorname{logit}(\text { theta })-\operatorname{logit}(\mathrm{rho} 0))^{*} X[\mathrm{i}]$

$\operatorname{logit}(\operatorname{eta}[\mathrm{i}, 3])<-\operatorname{logit}(\mathrm{rho} 0)+(1 / \mathrm{mtd})^{*}(\operatorname{logit}(\text { theta })-\operatorname{logit}(\mathrm{rho} 0))^{*} X[\mathrm{i}]$

$\#$ rho1 $=\mathrm{P}$ (grade 2 or more toxicity at initial dose $)$

$\#$ rho $0=\mathrm{P}($ DLT or grade 3 or 4 toxicity at initial dose $)$

\# MTD $=$ dose producing DLT in theta fraction of the population

for $(\mathrm{i}$ in $1: \mathrm{N})\{$

$\mathrm{p}[\mathrm{i}, 1]<-\mathrm{eta}[\mathrm{i}, 1]$

$\mathrm{p}[\mathrm{i}, 3]<-\operatorname{eta}[\mathrm{i}, 3]$

$\mathrm{p}[\mathrm{i}, 2]<-1-(\mathrm{eta}[\mathrm{i}, 1]+\mathrm{eta}[\mathrm{i}, 3])$

$\mathrm{Y}[\mathrm{i}] \sim \operatorname{dcat}(\mathrm{p}[\mathrm{i}]$,

\}

\# Prior Distributions

rho0 $\sim \operatorname{dunif}(0$, theta $)$

rho1 dunif(rho0, 1) 


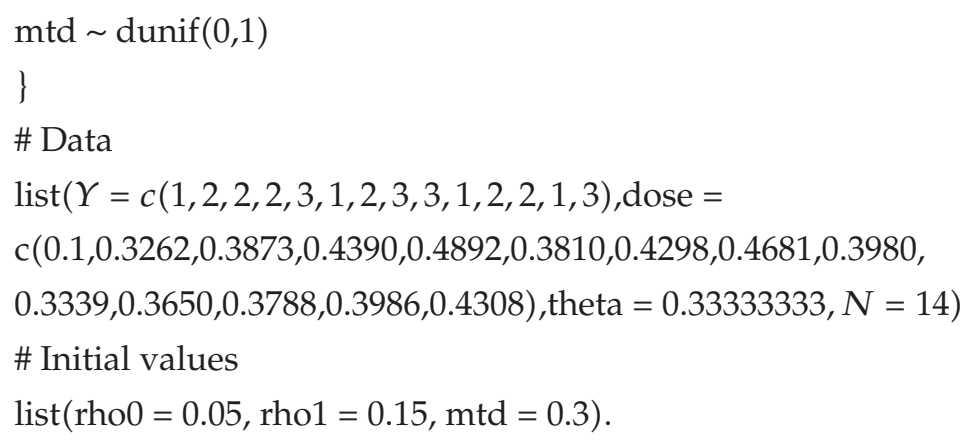

\section{Acknowledgments}

This paper supported in part by the National Center for Research Resources, Grant UL1RR033176, and is now at the National Center for Advancing Translational Sciences, Grant UL1TR000124 (M. Tighiouart and A. Rogatko), Grant 5P01CA098912-05 (A. Rogatko) and P01 DK046763 (A. Rogatko). The content is solely the responsibility of the authors and does not necessarily represent the official views of the NIH.

\section{References}

[1] T. G. Roberts Jr., B. H. Goulart, L. Squitieri et al., "Trends in the risk and benefits to patients with cancer participating in phase 1 clinical trials," Journal of the American Medical Association, vol. 292, no. 17, pp. 2130-2140, 2004.

[2] National Cancer Institute, Common Toxicity Criteria for Adverse Events v3.0 (CTCAE), 2003, http:// ctep.cancer.gov/reporting/ctc.html.

[3] J. O'Quigley, M. Pepe, and L. Fisher, "Continual reassessment method: a practical design for phase 1 clinical trials in cancer," Biometrics, vol. 46, no. 1, pp. 33-48, 1990.

[4] C. Gatsonis and J. B. Greenhouse, "Bayesian methods for phase I clinical trials," Statistics in Medicine, vol. 11, no. 10, pp. 1377-1389, 1992.

[5] S. D. Durham and N. Flournoy, "Random walks for quantile estimation," in Statistical Design Theory and Related Topics, pp. 467-476, Springer, New York, NY, USA, 1994.

[6] E. L. Korn, D. Midthune, T. T. Chen, L. V. Rubinstein, M. C. Christian, and R. M. Simon, "A comparison of two phase I trial designs," Statistics in Medicine, vol. 13, no. 18, pp. 1799-1806, 1994.

[7] J. Whitehead and H. Brunier, "Bayesian decision procedures for dose determining experiments," Statistics in Medicine, vol. 14, no. 9-10, pp. 885-893, 1995.

[8] J. Whitehead, "Bayesian decision procedures with application to dose-finding studies," International Journal of Pharmaceutical Medicine, vol. 11, no. 4, pp. 201-208, 1997.

[9] J. Babb, A. Rogatko, and S. Zacks, “Cancer phase I clinical trials: efficient dose escalation with overdose control," Statistics in Medicine, vol. 17, no. 10, pp. 1103-1120, 1998.

[10] M. Gasparini and J. Eisele, "A curve-free method for phase I clinical trials," Biometrics, vol. 56, no. 2, pp. 609-615, 2000.

[11] S. Mukhopadhyay, "Bayesian nonparametric inference on the dose level with specified response rate," Biometrics, vol. 56, no. 1, pp. 220-226, 2000.

[12] L. M. Haines, I. Perevozskaya, and W. F. Rosenberger, "Bayesian optimal designs for phase I clinical trials," Biometrics, vol. 59, no. 3, pp. 591-600, 2003.

[13] D. Faries, "Practical modifications of the continual reassessment method for phase I cancer clinical trials," Journal of Biopharmaceutical Statistics, vol. 4, no. 2, pp. 147-164, 1994.

[14] S. Moller, "An extension of the continual reassessment methods using a preliminary up-and-down design in a dose finding study in cancer patients, in order to investigate a greater range of doses," Statistics in Medicine, vol. 14, no. 9-10, pp. 911-922, 1995.

[15] S. N. Goodman, M. L. Zahurak, and S. Piantadosi, "Some practical improvements in the continual reassessment method for phase I studies," Statistics in Medicine, vol. 14, no. 11, pp. 1149-1161, 1995. 
[16] J. O'Quigley and L. Z. Shen, "Continual reassessment method: a likelihood approach," Biometrics, vol. 52, no. 2, pp. 673-684, 1996.

[17] S. Piantadosi, J. D. Fisher, and S. Grossman, "Practical implementation of a modified continual reassessment method for dose-finding trials," Cancer Chemotherapy and Pharmacology, vol. 41, no. 6, pp. 429-436, 1998.

[18] Y. K. Cheung and R. Chappell, "Sequential designs for phase I clinical trials with late-onset toxicities," Biometrics, vol. 56, no. 4, pp. 1177-1182, 2000.

[19] B. E. Storer, "An evaluation of phase I clinical trial designs in the continuous dose-response setting," Statistics in Medicine, vol. 20, no. 16, pp. 2399-2408, 2001.

[20] D. H. Y. Leung and Y. G. Wang, "An extension of the continual reassessment method using decision theory," Statistics in Medicine, vol. 21, no. 1, pp. 51-63, 2002.

[21] S. Zacks, A. Rogatko, and J. Babb, "Optimal Bayesian-feasible dose escalation for cancer phase I trials," Statistics $\mathcal{E}$ Probability Letters, vol. 38, no. 3, pp. 215-220, 1998.

[22] M. Tighiouart, A. Rogatko, and J. S. Babb, "Flexible Bayesian methods for cancer phase I clinical trials. Dose escalation with overdose control," Statistics in Medicine, vol. 24, no. 14, pp. 2183-2196, 2005.

[23] M. Tighiouart and A. Rogatko, "Dose finding with escalation with overdose control (EWOC) in cancer clinical trials," Statistical Science, vol. 25, no. 2, pp. 217-226, 2010.

[24] S. Lonial, J. Kaufman, M. Tighiouart et al., "A phase I/II trial combining high-dose melphalan and autologous transplant with bortezomib for multiple myeloma: a dose- and schedule-finding study," Clinical Cancer Research, vol. 16, no. 20, pp. 5079-5086, 2010.

[25] R. Sinha, J. L. Kaufman, H. J. Khouri, N. King, P. J. Shenoy, C. Lewis et al., "A phase 1 dose escalation study of bortezomib combined with rituximab, cyclophosphamide, doxorubicin, modified vincristine, and prednisone for untreated follicular lymphoma and other low-grade B-cell lymphomas," Cancer, vol. 118, no. 14, pp. 3538-3548, 2011.

[26] H. Borghaei, K. Alpaugh, G. Hedlund et al., "Phase I dose escalation, pharmacokinetic and pharmacodynamic study of naptumomab estafenatox alone in patients with advanced cancer and with docetaxel in patients with advanced non-small-cell lung cancer," Journal of Clinical Oncology, vol. 27, no. 25, pp. 4116-4123, 2009.

[27] J. D. Cheng, J. S. Babb, C. Langer et al., "Individualized patient dosing in phase I clinical trials: the role of escalation with overdose control in PNU-214936," Journal of Clinical Oncology, vol. 22, no. 4, pp. 602-609, 2004.

[28] R. J. Schilder, J. M. Gallo, M. M. Millenson et al., "Phase I trial of multiple cycles of high-dose carboplatin, paclitaxel, and topotecan with peripheral-blood stem-cell support as front-line therapy," Journal of Clinical Oncology, vol. 19, no. 4, pp. 1183-1194, 2001.

[29] G. M. Freedman, N. J. Meropol, E. R. Sigurdson et al., “Phase I trial of preoperative hypofractionated intensity-modulated radiotherapy with incorporated boost and oral capecitabine in locally advanced rectal cancer," International Journal of Radiation Oncology, Biology and Physics, vol. 67, no. 5, pp. 13891393, 2007.

[30] J. S. Babb and A. Rogatko, "Patient specific dosing in a cancer phase I clinical trial," Statistics in Medicine, vol. 20, no. 14, pp. 2079-2090, 2001.

[31] M. Tighiouart and A. Rogatko, “Dose escalation with overdose control," in Statistical Methods for DoseFinding Experiments, S. Chevret, Ed., pp. 173-188, John Wiley \& Sons, 2006.

[32] M. Tighiouart and A. Rogatko, "Dose finding in oncology-parametric methods," in Dose Finding in Drug Development, N. Ting, Ed., pp. 59-72, Springer, New York, NY, USA, 2006.

[33] A. Rogatko and M. Tighiouart, "Novel and efficient translational clinical trial designs in advanced prostate cancer," in Prostate Cancer, L. Chung, W. Isaacs, and J. W. Simons, Eds., pp. 487-495, Humana Press, New Jersey, NJ, USA, 2007.

[34] N. Ting, Dose Finding in Drug Development, Springer, New York, NY, USA, 1st edition, 2006.

[35] S. Chevret, Statistical Methods for Dose-Finding Experiments, John Wiley \& Sons, Chichester, UK, 2006.

[36] N. H. Gordon and J. K. V. Willson, "Using toxicity grades in the design and analysis of cancer phase I clinical trials," Statistics in Medicine, vol. 11, no. 16, pp. 2063-2075, 1992.

[37] C. Wang, T. T. Chen, and I. Tyan, "Designs for phase I cancer clinical trials with differentiation of graded toxicity," Communications in Statistics, vol. 29, no. 5-6, pp. 975-987, 2000.

[38] B. N. Bekele and P. F. Thall, "Dose-finding based on multiple toxicities in a soft tissue sarcoma trial," Journal of the American Statistical Association, vol. 99, no. 465, pp. 26-35, 2004.

[39] Z. Yuan, R. Chappell, and H. Bailey, "The continual reassessment method for multiple toxicity grades: a Bayesian quasi-likelihood approach," Biometrics, vol. 63, no. 1, pp. 173-179, 2007. 
[40] R. F. Potthoff and S. L. George, "Flexible phase I clinical trials: allowing for nonbinary toxicity response and removal of other common limitations," Statistics in Biopharmaceutical Research, vol. 1, no. 3, pp. 213-228, 2009.

[41] B. N. Bekele, Y. S. Li, and Y. A. Ji, "Risk-group-specific dose finding based on an average toxicity score," Biometrics, vol. 66, no. 2, pp. 541-548, 2010.

[42] E. M. Van Meter, E. Garrett-Mayer, and D. Bandyopadhyay, "Proportional odds model for dosefinding clinical trial designs with ordinal toxicity grading," Statistics in Medicine, vol. 30, no. 17, pp. 2070-2080, 2011.

[43] E. M. Van Meter, E. Garrett-Mayer, and D. Bandyopadhyay, “Dose-finding clinical trial design for ordinal toxicity grades using the continuation ratio model: an extension of the continual reassessment method," Clinical Trials, vol. 9, no. 3, pp. 303-313, 2012.

[44] A. Iasonos, S. Zohar, and J. O'Quigley, "Incorporating lower grade toxicity information into dose finding designs," Clinical Trials, vol. 8, no. 4, pp. 370-379, 2011.

[45] S. M. Lee, B. Cheng, and Y. K. Cheung, "Continual reassessment method with multiple toxicity constraints," Biostatistics, vol. 12, no. 2, pp. 386-398, 2011.

[46] Z. Chen, M. Tighiouart, and J. Kowalski, "Dose escalation with overdose control using a quasi-continuous toxicity score in cancer phase I clinical trials," Contemporary Clinical Trials, vol. 33, no. 5, pp. 949-958, 2012.

[47] N. Metropolis, A. W. Rosenbluth, M. N. Rosenbluth, A. H. Teller, and E. Teller, "Equation of state calculations by fast computing machines," Journal of Chemical Physics, vol. 21, no. 6, pp. 1087-1092, 1953.

[48] W. K. Hastings, "Monte carlo sampling methods using Markov chains and their applications," Biometrika, vol. 57, no. 1, pp. 97-109, 1970.

[49] D. J. Lunn, A. Thomas, N. Best, and D. Spiegelhalter, "WinBUGS—a Bayesian modelling framework: concepts, structure, and extensibility," Statistics and Computing, vol. 10, no. 4, pp. 325-337, 2000.

[50] Y. K. Cheung, "Coherence principles in dose-finding studies," Biometrika, vol. 92, no. 4, pp. 863-873, 2005. 


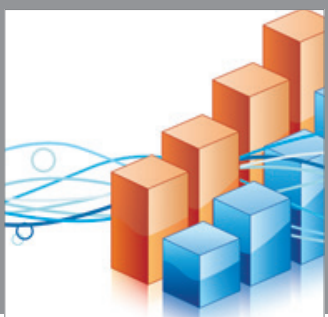

Advances in

Operations Research

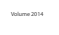

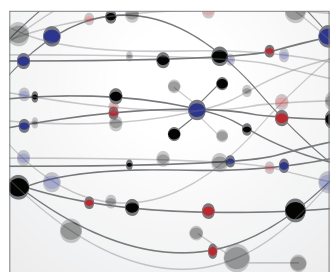

\section{The Scientific} World Journal
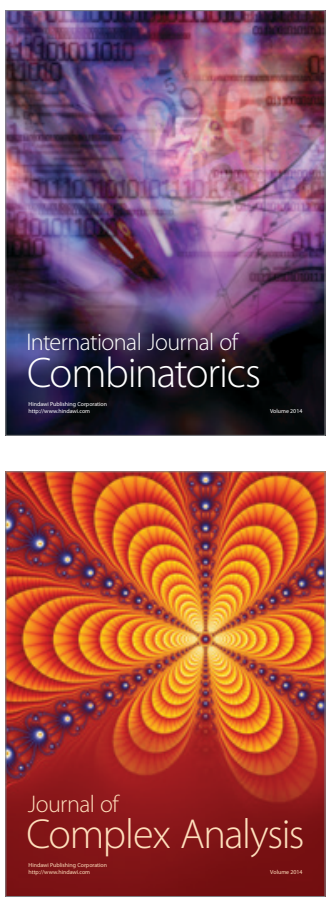

International Journal of

Mathematics and

Mathematical

Sciences
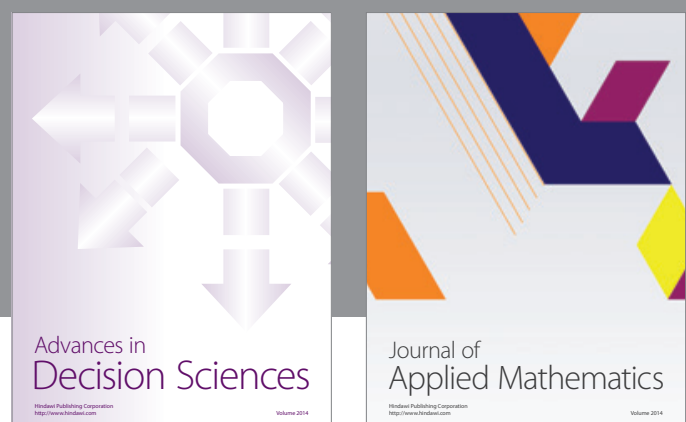

Journal of

Applied Mathematics
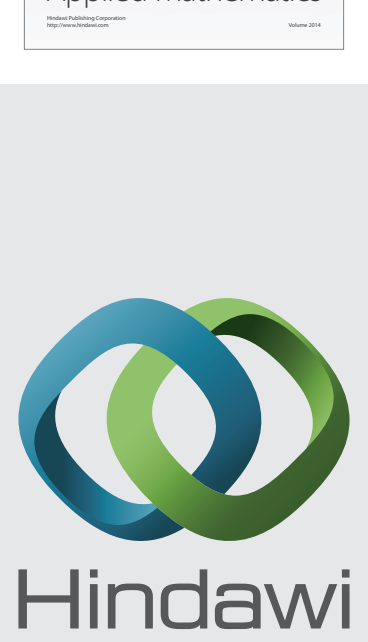

Submit your manuscripts at http://www.hindawi.com
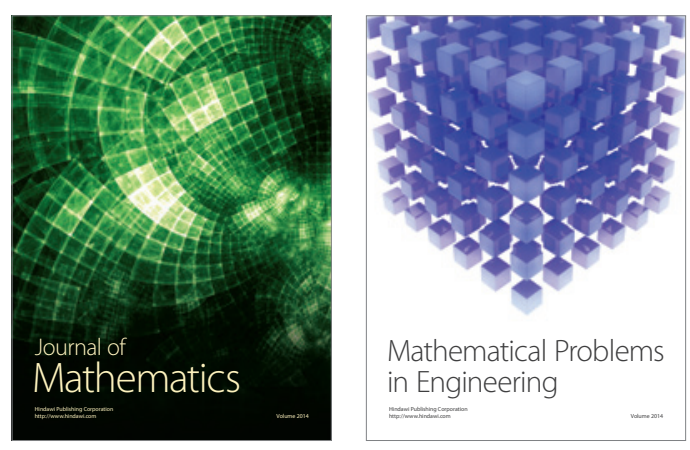

Mathematical Problems in Engineering
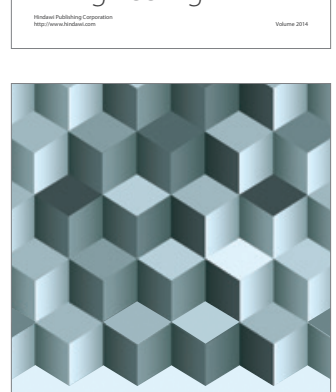

Journal of

Function Spaces
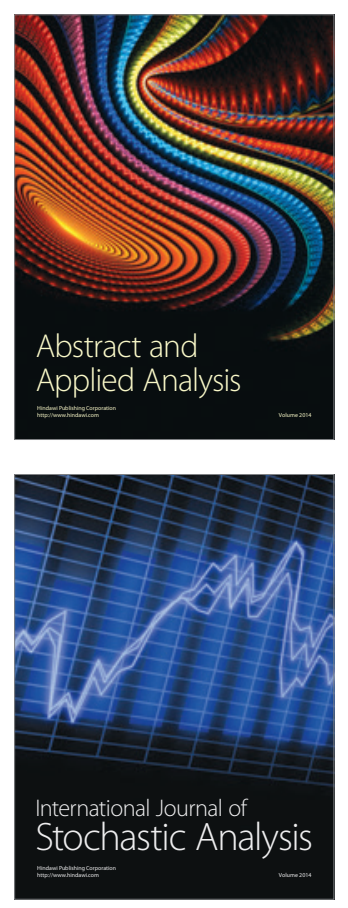

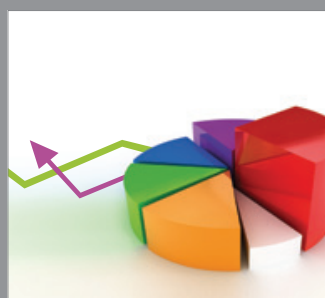

ournal of

Probability and Statistics

Promensencen
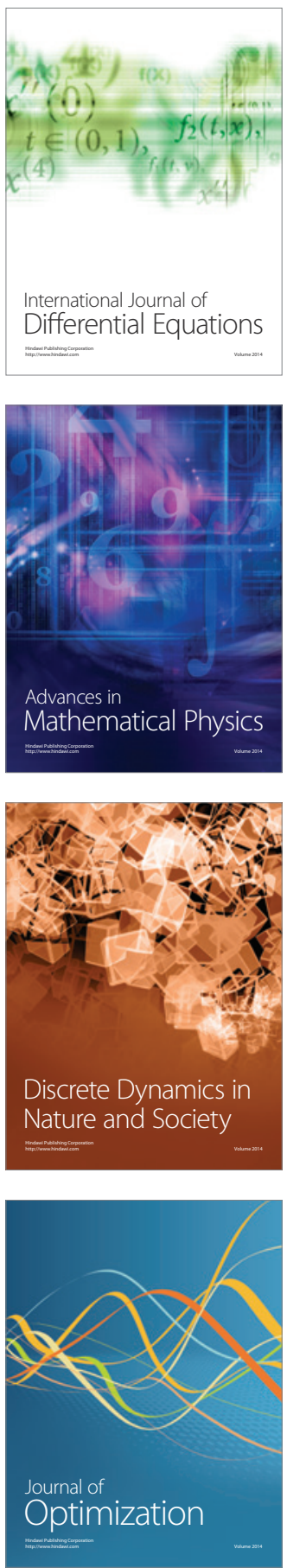